\title{
Noninvasive assessment of peripheral skeletal muscle weakness in idiopathic pulmonary fibrosis: a pilot study with multiparametric MRI of the rectus femoris muscle
}

\author{
Wagner Diniz de Paula, ${ }^{1}$ Marcelo Palmeira Rodrigues, ${ }^{2}$ Nathali Mireise Costa Ferreira, ${ }^{2}$ Viviane Vieira Passini, ${ }^{2}$ \\ César Augusto Melo-Silva ${ }^{2}$ \\ 'Department of Radiology, Brasilia University Hospital, University of Brasilia \\ ${ }^{2}$ Department of Pulmonology, Brasilia University Hospital, University of Brasilia, Brazil \\ Institution where the work was done: Hospital Universitário de Brasília / Universidade de Brasília, Brazil
}

\begin{abstract}
Background: To investigate differences in magnetic resonance imaging (MRI) features of rectus femoris muscle between idiopathic pulmonary fibrosis (IPF) patients and healthy volunteers.

Methods: Thirteen IPF patients with GAP Index stage II disease were subjected to pulmonary function tests, 6-minute walk test (6MWT), quadriceps femoris muscle strength measurement and MRI of the thigh at rest. At MRI, muscle cross-sectional areas, T2 and T2* relaxometry, and 3-point Dixon fat fraction were measured. The results were compared to those of eight healthy sedentary volunteers.

Results: IPF patients had significantly lower \%predicted FVC, $\mathrm{FEV}_{1}$ and $\mathrm{DL}_{\mathrm{CO}}(\mathrm{p}<0.001$ for the three variables) and walked significantly less in the 6MWT ( $\mathrm{p}=0.008)$. Mean quadriceps femoris muscle strength also was significantly lower in IPF patients $(\mathrm{p}=0.041)$. Rectus femoris muscle T2* measurements were significantly shorter in IPF patients $(\mathrm{p}=0.027)$. No significant intergroup difference was found regarding average muscle cross-sectional areas $(\mathrm{p}=0.790$ for quadriceps and $\mathrm{p}=0.816$ for rectus femoris $)$ or rectus femoris fat fraction $(\mathrm{p}=0.901)$. Rectus femoris T2 values showed a non-significant trend to be shorter in IPF patients $(\mathrm{p}=0.055)$.

Conclusions: Our preliminary findings suggest that, besides disuse atrophy, other factors such as hypoxia (but not inflammation) may play a role in the peripheral skeletal muscle dysfunction observed in IPF patients. This might impact the rehabilitation strategies for IPF patients and warrants further investigation.
\end{abstract}

Key words: Interstitial lung disease; idiopathic pulmonary fibrosis; skeletal muscle; magnetic resonance imaging.

Correspondence: Marcelo Palmeira Rodrigues, Department of Pulmonology, Brasilia University Hospital, University of Brasilia, SQSW 306, B, 603. 70.673-432 Brasilia, DF, Brazil. Tel. +55.61.984330879.

E-mail: pmarcelo27@gmail.com

Contributions: Each author has made substantial contribution to this paper and has approved the submitted version. WDP, wrote most part of the paper, discussed the conception and design, acquired imaging data and took part in analyses and interpretation; MPR, discussed the conception and design, acquired clinical data and took part in analyses and interpretation, and revised extensively the text; NMCF, discussed the conception and design, acquired clinical data and took part in analyses and interpretation; VVP, discussed the conception and design, acquired clinical data and took part in analyses and interpretation; CAM, discussed the conception and design, took part in analyses and interpretation, and revised extensively the text.

Conflict of interest: The authors declare that they have no competing interests in relation to this work.

Funding: None.

Ethics approval and consent to participate: This prospective study was approved by the local institutional review board and informed consent was obtained from all participants. Institution: University of Brasilia School of Medicine; Number: 701.077 (approved on June $6^{\text {th }}, 2014$ ). 


\section{Introduction}

Idiopathic pulmonary fibrosis (IPF) is a specific form of chronic, progressive, fibrosing interstitial pneumonia of unknown cause, which occurs primarily in older adults [1]. Limited to the lungs, it is the most common of the idiopathic interstitial pneumonias and carries the worst prognosis, with median survival ranging from 2.5 to 3.5 years prior to the advent of antifibrotic drugs [2]. Progressive dyspnea is the hallmark symptom and causes exercise limitation. Exertional dyspnea and exercise limitation lead to difficulties in performing daily activities and are major contributors to impairments in health-related quality of life [3].

The presence of exercise limitation and significant disability suggests that individuals with IPF are likely to be appropriate candidates for pulmonary rehabilitation. However, although there is evidence of short-term benefits [4,5], other investigators have shown that these gains are short-lived and only modest when compared to patients with chronic obstructive pulmonary disease (COPD) [3] and other interstitial lung diseases [6]. In COPD, reduced respiratory and lower extremity muscle strength are associated with decreased exercise and functional capacity, and muscle weakness is likely an important component of impairment and disability [7-9].

Previous research has shown that quadriceps femoris muscle weakness is related to exercise intolerance also in IPF $[9,10]$, but the underlying pathophysiology remains unknown. Possible mechanisms include deconditioning (disuse atrophy), decreased levels of anabolic hormones, an IPF-specific inflammatory myopathy, sarcopenia, hypoxemia, and oxidative stress, among others $[9,11,12]$.

Magnetic resonance imaging (MRI) can non-invasively detect pathological changes at the cellular and tissue level in skeletal muscle by probing different aspects of hydrogen nuclear magnetic properties that are affected by physiological and pathological features of the tissue [13]. Transverse magnetization relaxation time (T2) is increased in idiopathic inflammatory myopathies, and has been advocated to objectively gauge disease activity [14]. Transverse relaxation in gradient echo (GRE) pulse sequences is subject to bulk field dephasing effect caused by local magnetic field inhomogeneities, and its characteristic time is referred to as $\mathrm{T} 2 *$ relaxation. $\mathrm{T} 2 *$-weighted sequences are used to depict paramagnetic deoxyhemoglobin, methemoglobin, or hemosiderin in lesions and tissues [15]. Diffusion-weighted MRI is sensitive to molecular motion of water in tissues [13], and may reflect microscopic architectural changes in diseased muscle [16]. MRI also allows measurement of muscle volume [17,18], fat content $[18,19]$, and oxygenation [20,21]; and may provide valuable insight into muscle abnormalities observed in IPF patients.

The aim of our study was to investigate differences in morphologic and resting nuclear magnetic resonance properties of the rectus femoris muscle between patients with idiopathic pulmonary fibrosis and sedentary healthy controls.

\section{Design and Methods}

\section{Participants}

This prospective study was approved by our institutional review board and informed consent was obtained from all participants. From November 2016 to February 2019, we enrolled consecutive patients with IPF and healthy volunteers of comparable age, nonsmokers and not physically active.

Inclusion criteria in the study group were: diagnosis of idiopathic pulmonary fibrosis as per official guidelines in effect at the time of enrollment [22]; stage II disease based on the GAP (Gender, Age, lung Physiology) index [23]; and absence of other coexisting pulmonary diseases. Patients currently enrolled or previously subjected to pulmonary rehabilitation were excluded. We specified stage II disease (GAP index 4 or 5) in order to obtain a more homogeneous sample, devoid of mild or severe cases. Eight patients were being treated with pirfenidone and four with nintedanib, and one had not initiated antifibrotic treatment yet. No patients received corticosteroids.

As controls, we enrolled healthy volunteers with a sedentary lifestyle and no regular physical activity for at least six months, without respiratory symptoms or clinical diagnosis of any pulmonary disease, in the same age range of the IPF patients.

Musculoskeletal, neurological and cardiovascular disorders that might compromise locomotion or exercise capacity, including conditions known to cause sarcopenia, such as active malignancies, were regarded as exclusion criteria for both groups.

We recorded participants' age (yr), sex, body mass $(\mathrm{kg})$ and height $(\mathrm{m})$, and calculated the body mass index (BMI, defined as the body mass divided by the square of the body height), and the body surface area (BSA) [24].

\section{Functional characterization}

We documented the mMRC Dyspnea Scale [25] scores, the six-minute walk test [26] distances and the results of standardized pulmonary function tests [27], comprising spirometry and diffusing capacity for carbon monoxide $\left(\mathrm{DL}_{\mathrm{CO}}\right)$. Spirometric parameters recorded were forced vital capacity (FVC) and forced expiratory volume in one second $\left(\mathrm{FEV}_{1}\right)$, both absolute and as percentage of predicted for age and sex. The Tiffeneau-Pinelli index $\left(\mathrm{FEV}_{1} / \mathrm{FVC}\right.$ ratio) was then calculated and expressed as percentage. Quadriceps muscle strength was measured using maximal voluntary isometric contraction [28].

\section{MRI acquisition}

MR imaging was performed on a clinical closed-bore $1.5 \mathrm{~T}$ scanner with $33 \mathrm{mT} . \mathrm{m}^{-1}$ gradient and $120 \mathrm{~T} . \mathrm{m}^{-1} . \mathrm{s}^{-1}$ slew rate (Signa HDxt, GE Healthcare, Milwaukee MI, USA), with the participant in dorsal decubitus, using a phased-array 8-channel cardiac coil. Images of the right thigh were obtained in the transverse plane, at mid-femoral level (at $50 \%$ of femoral length), with $24 \mathrm{~cm}$ field of view and $5 \mathrm{~mm}$ slice thickness. Additional acquisition parameters are shown in Table 1.

Table 1. MRI pulse sequences and acquisition parameters.

$\begin{array}{lcccc}\text { Pulse sequence design } & \text { TR/TE }(\mathrm{ms}) & \text { FA/TI }\left({ }^{\circ} / \mathrm{ms}\right) & \begin{array}{c}\text { Matrix dimensions } \\ \text { (frequency } \mathbf{x} \text { phase) }\end{array} & \begin{array}{c}\text { Slice thickness / } \\ \text { interslice gap }(\mathrm{mm})\end{array} \\ \text { STIR } & 4000 / 38.9 & 180 / 90 / 150 & 320 \times 192 & 5 / 1 \\ \text { T2 Map } & 1000 / 7.4-59.1 & 90 /- & 320 \times 256 & 5 / 1 \\ \text { T2* Map } & 39 / 1.2-10.2 & 25 /- & 64 \times 64 & 5 / 1 \\ \text { IDEAL } & 10.6 / 6.0 & 10 /- & 256 \times 256 & 5 /-2.5\end{array}$

TR, repetition time; TE, echo time; FA, flip angle; TI, inversion time; STIR, short tau inversion recovery; IDEAL, iterative decomposition of water and fat with echo asymmetry and least-squares estimation. 
The fat-suppressed, T2-weighed STIR (short tau inversion recovery) pulse sequence is sensitive to free water and was used to visually assess imaging signs of muscle inflammation and damage [14]. We used fast multi-echo sequences for relaxometric quantification of $\mathrm{T} 2$ and $\mathrm{T} 2 *$ transverse magnetization times (T2 and T2* mapping). The T2 mapping pulse sequence was acquired with higher spatial resolution to be used also for morphological evaluation and muscle outlining. The IDEAL (Iterative Decomposition of water and fat with Echo Asymmetry and Least-squares estimation) sequence is a 3-point Dixon technique for water/fat separation and was used to compute muscle fat fraction [29].

Continuous composite Bézier regions of interest (ROI) outlining the rectus femoris muscle [12], quadriceps femoris muscle and thigh contour were manually drawn by a radiologist (WDP) with more than 10 years of experience, to obtain the respective absolute cross-sectional areas $\left(\mathrm{CSA}_{\mathrm{abs}}\right)$ on the central image in each series. To exclude variation in body size as a confounding factor, we performed allometric scaling of $\mathrm{CSA}_{\mathrm{abs}}$ using BSA, with a scaling exponent of $2 / 3\left(\mathrm{CSA}_{\text {corrected }}=\mathrm{CSA}_{\mathrm{abs}} \div \mathrm{BSA}^{2 / 3}\right) \cdot{ }^{30}$ For signal intensity (SI) measurements on T2 and T2* maps, we used the largest possible elliptical ROIs in the rectus femoris muscle, avoiding artifacts and excluding vessels and any other macroscopic non-muscle tissue. The rectus femoris muscle was selected for displaying the lowest coefficient of variation in a subsample of our SI measurements, for being readily separable from the remaining components of the quadriceps muscle, and for having been assessed in previous studies [12]. Fat fraction was calculated as the ratio of average SI on fat-only images and average SI on in-phase (water plus fat) images, expressed as percentage, in continuous composite Bézier ROI encompassing the entire cross-section of the rectus femoris muscle. Image analysis was performed with OsiriX MD (Pixmeo) and Advantage Windows Workstation FuncTool (GE Healthcare).

\section{Statistical analyses}

IBM SPSS Statistics for Mac version 26 (International Business Machines Corp.) was used for statistical analyses. Distribution of the interval and ratio variables were assessed with the Shapiro-Wilk normality test. Categorical variables were compared with Fisher's exact test. Interval and ratio variables were statistically compared using the t-test for independent samples or the independent-samples Mann-Whitey U test as appropriate. The tests were two-tailed and statistical significance was established at $\alpha<0.05$.

\section{Results}

The demographic and clinical variables are shown in Table 2. There was no intergroup difference in median age $(p=0.245)$, sex distribution $(\mathrm{p}=0.673)$, mean BMI $(\mathrm{p}=0.342)$ or mean BSA $(p=0.855)$. No participants in the control group complained of dyspnea (mMRC dyspnea scale score of zero) and all IPF patients had a non-zero mMRC score $(\mathrm{p}<0.001)$. The mean distance covered in the 6MWT was significantly shorter in the IPF group $(-32.1 \%$, $\mathrm{p}=0.008$ ), and the IPF patients also showed significantly lower mean quadriceps femoris muscle strength measurements than sedentary healthy controls $(-28 \%, \mathrm{p}=0.041)$. Participants in the IPF group had significantly lower mean \%predicted FVC $(-36 \%$, $\mathrm{p}<0.001), \operatorname{FEV}_{1}(-26 \%, \mathrm{p}<0.001)$ and $\mathrm{DL}_{\mathrm{CO}}(-52 \%, \mathrm{p}<0.001)$. Average FEV1/FVC ratio was significantly higher in IPF patients than in controls $(+9 \%, \mathrm{p}=0.027)$. Baseline $\mathrm{SpO}_{2}$ in the IPF group was $93.0 \% \pm 3.9$ (ranging from $84 \%$ to $98 \%$ ). During the $6 \mathrm{MWT}$, the $\mathrm{SpO}_{2}$ decreased from $92.8 \% \pm 3.1$ to $82.5 \% \pm 8.1$, with absolute decrease of $10.3 \% \pm 6.9(\mathrm{p}=0.004)$.

\section{MRI assessment}

Main results are shown in Table 3. No participant had overt muscle edema or otherwise visually abnormal muscle SI on STIR images. Intergroup difference in mean corrected cross-sectional muscle area was less than $3 \%$ and non-significant for both the quadriceps femoris muscle $(\mathrm{p}=0.790)$ and the rectus femoris muscle $(\mathrm{p}=0.816)$. Rectus femoris muscle $\mathrm{T} 2$ values ranged from 44.4 $\mathrm{ms}$ to $55.2 \mathrm{~ms}$ in the IPF group and from $47.9 \mathrm{~ms}$ to $59.6 \mathrm{~ms}$ in the control group, without reaching statistically significant difference in the mean value (lower in the IPF group by $6 \%, \mathrm{p}=0.055$ ). T2* values ranged from $25.2 \mathrm{~ms}$ to $33.2 \mathrm{~ms}$ in the IPF group and from $30.7 \mathrm{~ms}$ to $37.1 \mathrm{~ms}$ in the control group, with a significant difference in the mean value (lower in the IPF group by $8 \%, \mathrm{p}=0.027$ ). Figure 1 shows two examples of T2* maps. Fat fraction measurements ranged from $5.1 \%$ to $17.7 \%$ in the IPF group and from $5.6 \%$ to $10.3 \%$ in the control group, without significant intergroup difference $(\mathrm{p}=0.901)$.

Table 2. Demographics and clinical variables.

\begin{tabular}{|c|c|c|c|}
\hline & $\begin{array}{l}\text { IPF patients } \\
\qquad(n=13)\end{array}$ & $\begin{array}{l}\text { Control group } \\
(\mathrm{n}=8)\end{array}$ & $\mathbf{p}$ \\
\hline Age, yr & $77.0(66.0,81.5)$ & $67.0(65.3,73.3)$ & 0.245 \\
\hline Male & $8(62)$ & $4(50)$ & 0.673 \\
\hline BMI, kg.m ${ }^{-2}$ & $27.3 \pm 2.6$ & $29.5 \pm 6.0$ & 0.342 \\
\hline $\mathrm{BSA}, \mathrm{m}^{2}$ & $1.86 \pm 0.20$ & $1.90 \pm 0.31$ & 0.768 \\
\hline \multicolumn{4}{|l|}{ PFT } \\
\hline FVC, L & $2.22 \pm 0.81$ & $2.84 \pm 0.34$ & 0.025 \\
\hline FVC, \% predicted & $65.8 \pm 14.8$ & $102.9 \pm 12.3$ & $<0.001$ \\
\hline $\mathrm{FEV}_{1}, \mathrm{~L}$ & $1.90 \pm 0.56$ & $2.23 \pm 0.37$ & 0.155 \\
\hline $\mathrm{FEV}_{1}, \%$ predicted & $74.7 \pm 13.2$ & $101.0 \pm 8.7$ & $<0.001$ \\
\hline $\mathrm{FEV}_{1} / \mathrm{FVC}$ ratio, $\%$ & $84.8 \pm 6.7$ & $78.0 \pm 5.1$ & 0.027 \\
\hline $\mathrm{DL}_{\mathrm{CO}}, \mathrm{mmol} \mathrm{min}^{-1} \cdot \mathrm{kPa}^{-1}$ & $3.04 \pm 1.21$ & $5.99 \pm 0.69$ & $<0.001$ \\
\hline $\mathrm{DL}_{\mathrm{co}}, \%$ predicted & $38.6 \pm 8.7$ & $80.9 \pm 5.2$ & $<0.001$ \\
\hline Quadriceps strength, kgf & $30.9 \pm 13.1$ & $42.9 \pm 6.8$ & 0.041 \\
\hline 6MWT, m & $346 \pm 114$ & $510 \pm 49$ & 0.008 \\
\hline mMRC score $(0 / 1 / 2 / 3)$ & $0 / 3 / 2 / 8$ & $8 / 0 / 0 / 0$ & $<0.001$ \\
\hline
\end{tabular}

IPF, idiopathic pulmonary fibrosis; BMI, body mass index; BSA, body surface area; PFT, pulmonary function tests; $\mathrm{FVC}$, forced vital capacity; $\mathrm{DL}_{\mathrm{CO}}$, diffusion capacity for carbon monoxide; $\mathrm{FEV}_{1}$ forced expiratory volume in one second; 6MWT, six-minute walk test; mMRC, modified Medical Research Council Dyspnea Scale. Data are presented as mean \pm SD for normally distributed data median (interquartile range) for non-normally distributed data, or n (\%) for categorical data.

Table 3. Comparison of groups for MRI assessment.

\begin{tabular}{lccc} 
& $\begin{array}{c}\text { IPF patients } \\
(\mathbf{n = 1 3})\end{array}$ & $\begin{array}{c}\text { Control group } \\
(\mathbf{n}=\mathbf{8})\end{array}$ & $\mathbf{p}$ \\
$\begin{array}{l}\text { Quadriceps muscle } \\
\text { cross-sectional area }\end{array}$ & & \\
$\quad$ Absolute, $\mathrm{cm}^{2}$ & & & \\
$\quad$ Corrected, $\mathrm{cm}^{2} . \mathrm{m}^{-2}$ & $49.0(45.5,59.0)$ & $43.5(39.0,71.5)$ & 0.737 \\
Rectus femoris muscle & $33.7 \pm 6.1$ & $34.6 \pm 8.6$ & 0.790 \\
cross-sectional area & & & \\
$\quad$ Absolute, $\mathrm{cm}^{2}$ & & & \\
$\quad$ Corrected, cm ${ }^{2} \mathrm{~m}^{-2}$ & $5.3 \pm 1.7$ & $5.5 \pm 2.1$ & 0.762 \\
Rectus femoris muscle & $3.5 \pm 1.0$ & $3.6 \pm 1.1$ & 0.816 \\
SI measurements & & & \\
$\quad$ T2, ms & & & \\
$\quad$ T2*, ms & $49.3 \pm 2.8$ & $52.6 \pm 4.4$ & 0.055 \\
$\quad$ Fat fraction, \% & $30.2 \pm 2.5$ & $32.7 \pm 2.0$ & 0.027 \\
\hline
\end{tabular}

IPF, idiopathic pulmonary fibrosis; SI, signal intensity. Data are presented as mean \pm SD for normally distributed data, or median (interquartile range) for non-normally distributed data. 


\section{Discussion}

Muscle dysfunction may be assessed through a variety of invasive and non-invasive methods. Muscle strength can be measured by means of isokinetic or isometric dynamometry, but it does not provide insight into pathophysiological mechanisms. Muscle structure and composition can be directly examined by tissue sampling techniques, but their invasive nature and low availability hinder their widespread use. Imaging modalities allow non-invasive evaluation of morphological aspects, including volume-related measurements (e.g., cross-sectional area and thickness), and presence and degree of myosteatosis. MRI, in particular, can also extract metabolism-related features. To the best of our knowledge, this is the first study to address MRI-specific features of skeletal muscle in IPF patients.

We found significantly shorter $\mathrm{T} 2 *$ values in the rectus femoris muscle of IPF patients compared to sedentary healthy controls. Shorter T2* values suggest larger local magnetic field inhomogeneities, and we hypothesize this could be related to higher concentration of paramagnetic substances in skeletal muscle tissue, such as deoxyhemoglobin or deoxymyoglobin [31-33]. Whether this is in fact the case remains an open question, and further investigation is warranted to directly evaluate the potential role of an ischemic/hypoxic factor in the pathogenesis of the skeletal muscle dysfunction observed in IPF patients. This could not only improve our understanding of the mechanisms underlying the impaired response to rehabilitation in this condition but also ultimately modify therapeutic approaches.

We found neither visual signs of muscle edema nor longer T2 values in IPF patients, arguing against the assumption of a diffuse inflammatory myopathy in IPF. In fact, T2 values tended to be lower in the IPF group, albeit not reaching statistical significance. Muscle cross-sectional area and fat fraction in IPF patients were also not statistically different from sedentary healthy controls, findings that are in line with the role of physical inactivity in exercise intolerance, but the significant intergroup difference in quadriceps strength measurements supports the notion of a multifactorial nature of muscle dysfunction in IPF [34], as one would expect a greater degree of muscle atrophy or myosteatosis should disuse atrophy being the sole causative mechanism. As expected, pulmonary compliance was lower in IPF patients, as evinced by the significantly lower FVC values in this group. The preferential decrease of FVC over $\mathrm{FEV}_{1}$ accounts for the significantly increased Tiffeneau-Pinelli index in IPF patients, a marker of disease severity. The substantially reduced pulmonary diffusing capacity in the IPF group was also anticipated. Both pulmonary functional abnormalities and quadriceps muscle weakness may contribute to the observed intergroup difference in 6MWT performance.

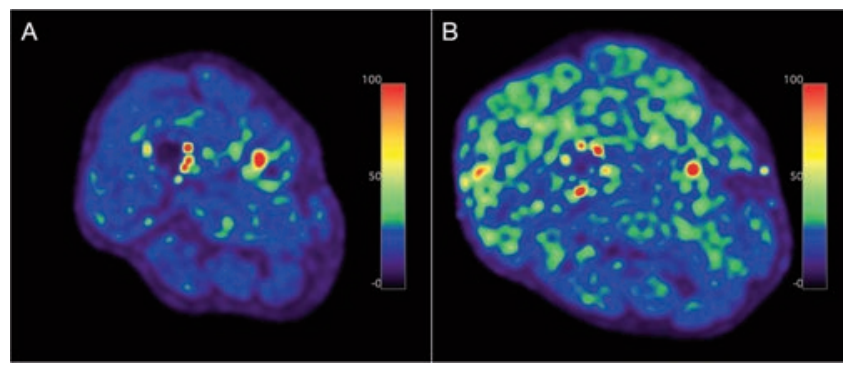

Figure 1. T2* maps in a patient with idiopathic pulmonary fibrosis (A) and in a sedentary healthy volunteer (B).
Our work had some limitations. Owing to the prospective nature of the study, the sample size was relatively small, being especially difficult to enroll healthy participants with a sedentary lifestyle in the age range of the IPF patients. The small sample size limits the power of the study, so small differences may have remained uncovered. Besides, the small sample size also precluded the employment of correlation analysis. Furthermore, due to the cross-sectional design of the study, we did not evaluate the effect of rehabilitation on the measured MRI parameters. Lastly, some derangements in skeletal muscle may not be apparent at rest, requiring exercise or ischemia to be disclosed.

\section{Conclusions}

This pilot study showed significantly shorter $\mathrm{T} 2 *$ in the rectus femoris muscle of IPF patients at rest, without significant difference in T2, fat fraction or cross-sectional area, in comparison with sedentary healthy volunteers. These findings support the notion that disuse atrophy is involved in the skeletal muscle dysfunction observed in IPF, but in addition suggest that other factors, such as hypoxia, may also play a role, without evidence of an inflammatory component. This may have potential clinical value in the rehabilitation of IPF patients and warrants further investigation.

\section{References}

1. Raghu G, Remy-Jardin M, Myers JL, Richeldi L, Ryerson CJ, Lederer DJ, et al. Diagnosis of idiopathic pulmonary fibrosis: An official ATS/ERS/JRS/ALAT clinical practice guideline. Am J Respir Crit Care Med 2018;198:e44-68.

2. Ley B, Collard HR, King Jr TE. Clinical course and prediction of survival in idiopathic pulmonary fibrosis. Am J Respir Crit Care Med 2011;183:431-40.

3. Kozu R, Senjyu H, Jenkins SC, Mukae H, Sakamoto N, Kohno $\mathrm{S}$. Differences in response to pulmonary rehabilitation in idiopathic pulmonary fibrosis and chronic obstructive pulmonary disease. Respiration 2011;81:196-205.

4. Swigris JJ, Fairclough DL, Morrison M, Make B, Kozora E, Brown KK, et al. Benefits of pulmonary rehabilitation in idiopathic pulmonary fibrosis. Respir Care 2011;56:783-9.

5. Dowman L, Hill CJ, Holland AE. Pulmonary rehabilitation for interstitial lung disease. Cochrane Database Syst Rev 2014;10:CD006322.

6. Holland AE, Hill CJ, Glaspole I, Goh N, McDonald CF. Predictors of benefit following pulmonary rehabilitation for interstitial lung disease. Respir Med 2012;106:429-35.

7. Singer J, Yelin EH, Katz PP, Sanchez G, Iribarren C, Eisner $\mathrm{MD}$, et al. Respiratory and skeletal muscle strength in chronic obstructive pulmonary disease. J Cardiopulm Rehabil Prev 2011;31:111-9.

8. Leite Rodrigues S, Melo e Silva CA, Lima T, de Assis Viegas CA, Palmeira Rodrigues M, Almeida Ribeiro F. The influence of lung function and muscular strength on the functional capacity of chronic obstructive pulmonary disease patients. Rev Port Pneumol 2009;15:199-214.

9. Nishiyama O, Taniguchi H, Kondoh Y, Kimura T, Ogawa T, Watanabe F, et al. Quadriceps weakness is related to exercise capacity in idiopathic pulmonary fibrosis. Chest 2005;127: 2028-33.

10. Nolan CM, Kon SSC, Canavan JL, Jones SE, Maddocks M, Cullinan P, et al. Preferential lower limb muscle weakness in idiopathic pulmonary fibrosis: Effects on exercise capacity. 
Eur Respir J 2014;44:P4492.

11. Moon S, Choi J, Lee S, Jung KS, Jung JY, Kang YA, et al. Thoracic skeletal muscle quantification: low muscle mass is related with worse prognosis in idiopathic pulmonary fibrosis patients. Respir Res 2019;20:35.

12. Mendes P, Wickerson L, Helm D, Janaudis-Ferreira T, Brooks $\mathrm{D}$, Singer LG, et al. Skeletal muscle atrophy in advanced interstitial lung disease. Respirology 2015;20:953-9.

13. Feng S, Chen D, Kushmerick M, Lee D. Multiparameter MRI analysis of the time course of induced muscle damage and regeneration. J Magn Reson Imaging 2014;40:779-88.

14. Yao L, Yip A, Shrader JA, Mesdaghinia S, Volochayev R, Jansen AV, et al. Magnetic resonance measurement of muscle $\mathrm{T} 2$, fat-corrected T2 and fat fraction in the assessment of idiopathic inflammatory myopathies. Rheumatology (Oxford) 2016;55:441-9.

15. Chavhan GB, Babyn PS, Thomas B, Shroff MM, Haacke EM. Principles, techniques, and applications of T2*-based MR imaging and its special applications. RadioGraphics 2009;29:1433-49.

16. Carlier PG, Marty B, Scheidegger O, Loureiro de Sousa P, Baudin PY, Snezhko E, et al. Skeletal muscle quantitative nuclear magnetic resonance imaging and spectroscopy as an outcome measure for clinical trials. J Neuromuscul Dis 2016; $3: 1-28$

17. Mandic M, Rullmann E, Widholm P, Lilja M, Dahlqvist Leinhard O, Gustafsson T, et al. Automated assessment of regional muscle volume and hypertrophy using MRI. Sci Rep 2020;10:2239.

18. Kälin PS, Crawford RJ, Marcon M, Manoliu A, Bouaicha S, Fischer MA, et al. Shoulder muscle volume and fat content in healthy adult volunteers: quantification with DIXON MRI to determine the influence of demographics and handedness. Skeletal Radiol 2018;47:1393-402.

19. Inhuber S, Sollmann N, Schlaeger S, Dieckmeyer M, Burian E, Kohlmeyer C, et al. Associations of thigh muscle fat infiltration with isometric strength measurements based on chemical shift encoding-based water-fat magnetic resonance imaging. Eur Radiol Exp 2019;3:45.

20. Klarhöfer M, Madörin P, Bilecen D, Scheffler K. Assessment of muscle oxygenation with balanced SSFP: A quantitative signal analysis. J Magn Reson Imaging 2008;27:1169-74.

21. Partovi S, Schulte AC, Jacobi B, Klarhöfer M, Lumsden AB, Loebe $\mathrm{M}$, et al. Blood oxygenation level-dependent (BOLD) MRI of human skeletal muscle at 1.5 and 3 T. J Magn Reson Imaging. 2012;35:1227-1232.

22. Raghu G, Collard HR, Egan JJ, Martinez FJ, Behr J, Brown $\mathrm{KK}$, et al. An official ATS/ERS/JRS/ALAT statement: Idiopathic pulmonary fibrosis: Evidence-based guidelines for diagnosis and management. Am J Respir Crit Care Med
2011;183:788-824.

23. Ley B, Ryerson CJ, Vittinghoff E, Ryu JH, Tomassetti S, Lee JS, et al. A multidimensional index and staging system for idiopathic pulmonary fibrosis. Ann Intern Med 2012;156:684-91.

24. Mosteller RD. Simplified calculation of body surface area. N Engl J Med 1987;317:1098.

25. Launois C, Barbe C, Bertin E, Nardi J, Perotin JM, Dury S, et al. The modified Medical Research Council scale for the assessment of dyspnea in daily living in obesity: a pilot study. BMC Pulm Med 2012;12:61.

26. Holland AE, Spruit MA, Troosters T, Puhan MA, Pepin V, Saey D, et al. An official European Respiratory Society/American Thoracic Society technical standard: field walking tests in chronic respiratory disease. Eur Respir J 2014;44:1428-46.

27. Miller MR, Hankinson J, Brusasco V et al. Standardisation of spirometry. Eur Respir J 2005;26:319-38.

28. Rodrigues SL, Melo-Silva CA, Lima T, Viegas CAA, Rodrigues MP, Ribeiro FA. The influence of lung function and muscular strength on the functional capacity of chronic obstructive pulmonary disease patients. Rev Port Pneumol 2009;15:199-214.

29. Reeder SB, Pineda AR, Wen Z, Shimakawa A, Yu H, Brittain $\mathrm{JH}$, et al. Iterative decomposition of water and fat with echo asymmetry and least-squares estimation (IDEAL): application with fast spin-echo imaging. Magn Reson Med 2005;54:63644.

30. Pan J, Stehling C, Muller-Hocker C, Schwaiger BJ, Lynch J, McCulloch CE, et al. Vastus lateralis/vastus medialis crosssectional area ratio impacts presence and degree of knee joint abnormalities and cartilage T2 determined with 3T MRI - an analysis from the incidence cohort of the Osteoarthritis Initiative. Osteoarthritis Cartilage 2011;19:65-73.

31. Kreutzer U, Wang DS, Jue T. Observing the 1H NMR signal of the myoglobin Val-E11 in myocardium: An index of cellular oxygenation. Proc Natl Acad Sci USA 1992;89:4731-3.

32. Kreis R, Bruegger K, Skjelsvik C, Zwicky S, Ith M, Jung B, et al. Quantitative $1 \mathrm{H}$ magnetic resonance spectroscopy of myoglobin de- and reoxygenation in skeletal muscle: Reproducibility and effects of location and disease. Magn Reson Med 2001;46:240-8.

33. Mancini DM, Wilson JR, Bolinger L, Li H, Kendrick K, Chance B, Leigh JS. In vivo magnetic resonance spectroscopy measurement of deoxymyoglobin during exercise in patients with heart failure. Demonstration of abnormal muscle metabolism despite adequate oxygenation. Circulation 1994;90:500-8.

34. Panagiotou M, Polychronopoulos V, Strange C. Respiratory and lower limb muscle function in interstitial lung disease. Chron Respir Dis 2016;13:162-72.

Received for publication: 3 August 2020. Accepted for publication: 13 October 2020.

This work is licensed under a Creative Commons Attribution-NonCommercial 4.0 International License (CC BY-NC 4.0).

(C) Copyright: the Author(s), 2020

Licensee PAGEPress, Italy

Multidisciplinary Respiratory Medicine 2020; 15:707

doi:10.4081/mrm.2020.707 